\title{
Le théâtre provocateur de Nicoleta Esinencu, dramaturge « Dans ta gueule »
}

\section{Véronique Boutin}

\section{(2) OpenEdition \\ Journals}

Édition électronique

URL : http://journals.openedition.org/lcc/592

DOI : $10.4000 /$ lcc.592

ISSN : 2430-4247

\section{Éditeur}

Université Aix-Marseille (AMU)

\section{Référence électronique}

Véronique Boutin, «Le théâtre provocateur de Nicoleta Esinencu, dramaturge « Dans ta gueule » », Les chantiers de la création [En ligne], 6 | 2013, mis en ligne le 15 janvier 2015, consulté le 08 avril 2020. URL : http://journals.openedition.org/lcc/592 ; DOI : https://doi.org/10.4000/lcc.592

Ce document a été généré automatiquement le 8 avril 2020.

Tous droits réservés 


\title{
Le théâtre provocateur de Nicoleta Esinencu, dramaturge « Dans ta gueule »
}

\author{
Véronique Boutin
}

1 Parler d'un âge d'or de la provocation dans le domaine du théâtre, c'est évoquer inévitablement le In-yer-face drama, mouvement britannique (et américain) des années 1980-1990, et avant lui, les pièces du Kitchen-sink theater des années cinquante, dont les personnages de milieux populaires, angry young men, vivaient une réalité sociale déprimante. Ce théâtre avait pour objectif de choquer, de faire réagir le public; on l'associe à un langage cru, à des scènes de violence et de sexe. Cette provocation n'était bien entendu pas gratuite: le In-yer-face est un théâtre politique; des pièces percutantes telles Shopping and Fucking de Mark Ravenhill ou Blasted de Sarah Kane ont pour volonté première de proposer une critique incisive de la vie moderne, de ses phénomènes de violence, du mythe du post-féminisme et de la société de consommation.

2 A l'aube des années 2000, après une fin de siècle riche en événements, dont la chute du Mur de Berlin, la fin du communisme, les guerres balkaniques, les critiques remarquent que les écrivains de théâtre (européens ou non) se rejoignent de nouveau dans une volonté d'écriture politique et sociale. On remarque parmi eux enfin bon nombre d'auteurs des pays d'Europe centrale et orientale: les Macédoniens Dukovski et Stefanovski, les Roumain(e)s Carbunariu, Peca et Stănescu, la Serbe Biljana Srbljanovic accouchent d'un théâtre radical. Le metteur en scène Miloš Lazin regroupe ces dramaturges barbares et autres dans le «Nouveau drame» («Nove drama»); Dominique Dolmieu, directeur de la Maison d'Europe et d'Orient, est quant à lui l'initiateur d'une appellation directement traduite du In-yer-face drama: le théâtre « Dans ta gueule».

Peut-on parler d'une filiation réfléchie du « Dans ta gueule » au In-yer-face? Non, en ce qui concerne les dramaturges de l'ancien bloc soviétique qui nous intéressent. Comme nous le précise Mirella Patureau, « le « jeune théâtre roumain » naît après 2002 (voir le 
groupe presque mythique DramAcum autour de Gianina Carbunariu, auquel s'est rajoutée plus tard Esinencu) ; dans les années 90, ils étaient la plupart à la maternelle " et ils n'ont pas eu accès alors aux textes du In-yer-face, mais « dès 2004, ils sont presque tous allés au Royal Court un peu comme à la Mecque.» Leur rage d'écrire un théâtre charnel, violent, pornographique, au langage sexuel et provocateur, leur sort donc simplement des tripes, du vécu d'une génération de jeunes artistes lucides, désireux d'exprimer un ras-le-bol politique face à la misère sociale dans le post-communisme.

La Moldave Nicoleta Esinencu est l'une de ces dramaturges enragées et engagées du «Dans ta gueule ». Ce sont les motifs et les objectifs de son écriture provocante que je veux tenter de mettre à plat à partir de trois de ses monologues : FUCK YOU, Eu.ro.Pa!, A(II) Rh+ et Mères sans chatte. Nous ausculterons l'art du titre et le langage virulent de cette auteur ; nous envisagerons ensuite les cibles de cette nouvelle angry young girl, qui s'inscrit dans un théâtre social et réaliste, dans la contemporanéité violente de la politique et moldave et européenne. Nous comprendrons enfin que si la dramaturge moldave pro-voque, étymologiquement appelle " en avant » ses compatriotes et suscite en eux une réaction émotionnelle, c'est dans le but de les amener à un acte créateur qui les reconstruirait politiquement.

\section{La façade provocatrice de Nicoleta Esinencu}

Parmi les trois monologues traités, la pièce FUCK YOU, Eu.ro.Pa!, écrite en Allemagne fin 2003, a le titre le plus accrocheur. L'injonction en anglais est universellement comprise. Le titre direct, explicite, sexuel et violent, en majuscules agressives doublées d'un point d'exclamation revanchard, qui ordonne à l'Europe d'aller se faire " enculer », a suscité une controverse en Roumanie en 2005, pays qui n'avait alors pas encore intégré l'Union Européenne. Le texte avait été publié pour le pavillon roumain lors de la Biennale de Venise; les représentations en ont été interdites. Esinencu a dû titrer: Stop Europe! pour qu'elles reprennent. Elle nous confia en 2010 qu'« il était plus important de jouer à Chisinau que d'avoir un casus belli avec le ministère. »

L'autre titre vulgaire Mères sans chatte affiche aussi un langage cru et une connotation sexuelle. La traductrice Mirella Patureau de Mame fara pizda nous indique que «le mot « pizda » en roumain qui désigne le sexe féminin est très vulgaire, assez ordurier. Je ne lui connais pas d'acception plus neutre. Quand j'ai traduit ce monologue, au début j'étais un peu réticente, car c'est ma langue maternelle et je sentais la violence du mot. J'ai proposé « vagin », mais Nicoleta a refusé et elle a eu raison. »

7 Esinencu provoque et à l'orée et dans le texte en utilisant un langage putassier. Mères sans chatte évoque la relation d'une gamine avec sa mère et sa grand-mère qui la traite de "pute » (1). La pièce met en avant le tabou de la sexualité féminine et s'achève par un «mazarfack!» (15) bien senti. Dans FUCK YOU, Eu.ro.Pa!, le personnage féminin imagine un dialogue avec un vendeur de merde et l'agresse: "J'aimerais chier avec plaisir sur toute votre Europe !» (34).

Nicoleta Esinencu emploie ce langage grossier pour plusieurs raisons : elle donne la parole à des personnages très jeunes et en colère qui utilisent un langage exutoire ; sans entrer en dissidence, elle est libre de tout dire depuis la fin du communisme, de la censure et du chaste réalisme socialiste ; enfin, elle choisit d'agresser et provoque parce que c'est un moyen pour elle de se faire entendre. Esinencu n'est ni punk, ni anarchiste : son langage est avant tout révélateur d'une violence quotidienne, familiale 
et sociale dont elle est témoin et dont elle témoigne. Qui plus est, dans son théâtre "Dans ta gueule », plus que son vocabulaire, ce sont les situations qu'elle dépeint qui sont fortes et mettent à mal le public pour lui désigner ses exactions.

\section{Une critique acerbe du monde moldave et européen}

Esinencu est considérée comme provocatrice par ses concitoyens, parce qu'elle ne porte pas sur eux un regard compatissant. La Moldavie reste le pays le plus pauvre d'Europe, un pays économiquement attardé, politiquement en faille, avec un gouvernement quasi inexistant. Esinencu est une dramaturge lucide, qui propose dans un théâtre speculum un état des lieux et de sa population: elle happe, vitupère, malmène, interpelle les Moldaves d'hier et d'aujourd'hui.

Esinencu écrit un théâtre frontal : les trois textes ciblés sont des monologues familiaux où le personnage sur scène s'exprime en une adresse directe au public, ce qui fait que celui-ci se sent observé, concerné, voire visé. Dans FUCK YOU, Eu.ro.Pa!, une jeune fille s'adresse à son père et le spectateur devient ce «tu » et endosse le rôle du père; dans $A(I I) R h+$, le personnage s'adresse à lui-même à la deuxième personne du singulier. Ce « tu »-là est encore plus pernicieux, car le comédien le balance au public qui endosse le rôle du parricide.

11 Mais quels vices dénonce la provocatrice Esinencu qui ne fait que peindre une vérité familiale et sociale glauque et terrible, à moins qu'elle ne l'exagère pour alerter ses contemporains sur leurs dérives? En résumé, la dramaturge met sur scène des alcooliques, des pédophiles, des lâches, des demeurés, des loosers attardés, des parricides.

12 Dans FUCK YOU, Eu.ro.Pa!, le personnage féminin, porte-parole de sa génération ${ }^{1}$, règle ses comptes avec son père, avec son pays, avec le communisme et la pauvreté qui lui faisait manger des tartines de dentifrice, et avec un peuple par trop soumis :

Qu'est-ce qu'il a fait mon pays pour moi ? Papa, je n'aime pas qu'on m'encule. Cela me rappelle la Patrie [...] Les gens ici, Papa, [...] ne s'imaginent pas la vie sans souffrance. Tout leur fait mal. La tête. La gorge. L'estomac. Le cœur. L'appendice. Les reins. Les poumons. Mais le plus souvent, le foie. Non, papa, pas le foie, les genoux ! [...] Des martyrs! Et si le sort [...] ne leur offre pas l'occasion de vivre un drame, ils l'inventent. (24)

13 Le peuple moldave métaphoriquement sodomite a subi le communisme sans broncher et subit le capitalisme de la même manière : «Voilà, papa, les lettres que je reçois de vous... Presque rien de neuf. Aujourd'hui on va acheter deux sacs de pommes de terre pour avoir de quoi manger.» (29) L'image moldave est encore égratignée dans cette pièce revancharde lorsque le personnage évoque ce qu'elle en lit dans les journaux: «Un petit-fils de quatorze ans a baisé sa grand-mère de quatre-vingts ans. Un père a tué son fils en le frappant à la tête avec une hache. Des suicides en série. Un neuvième cas de suicide cette semaine. Une jeune fille de quatorze ans s'est jetée du septième étage. Sur sa poitrine était écrit «tATu » avec du rouge à lèvres. » (30)

L'alcoolisme, le racisme et le machisme sont autant de tares visées cette fois dans la pièce $A(I I) R h^{+}$, qui relate le parcours d'un père xénophobe qui bat sa fillette à mort parce qu'il imagine, dans son ivresse, qu'un jour elle pourrait fréquenter un Noir et il refuse qu'on lui injecte le sang d'un étranger qui pourrait la sauver. La violence de la scène est renforcée par le pronom personnel «tu » évoqué plus haut et qui vaut pour 
acte d'accusation au public en place : «tu rentres à la maison et tu demandes à ta fille de fermer toutes les fenêtres et après avoir bu tes protéines tu enlèves ta ceinture et tu la cognes pour que tout ceci n'arrive jamais et pendant que tu la frappes tu cries vers ta femme je propose de trouver un pays de payer un pays [...] où tous ceux qui ne sont pas de notre sang émigrent ». (24)

Un autre sujet central abordé dans les trois pièces par la dramaturge est l'hypocrisie face à la question de la sexualité dans un pays rétrograde. Le corps et ses besoins scatologiques et sexuels sont tabous, donc sont niés. La sexualité féminine surtout est masquée, hypocrisie dénoncée par Esinencu dans Mères sans chatte lorsque la fillette raconte à sa mère que le policier de quartier a «mis sa langue dans [sa] bouche et sa main dans [sa] culotte » (5). Ses cours d'éducation sexuelle sont élidés : «ma camarade d'école m'a dit si tu n'es pas complètement nue tu ne tombes pas enceinte et la prof de biologie a dit que le paragraphe 42 on va le lire toutes seules à la maison et comment il s'appelle le paragraphe 42 ? ne fais pas la maligne, compris?» (9) La mère ordonne alors à sa fille trop curieuse de porter un foulard sur les yeux tous les jours pendant quinze minutes. L'humour de Nicoleta Esinencu dans cette censure sexuelle nous donne à voir encore le procès de la famille de l'appart 148 parce que la mère a mis à sécher dehors « la chose intime » (4): ses petites culottes; et alors que la fillette a énuméré la soi-disant pudeur des femmes de son entourage, elle nous apprend finalement que toutes ont été arrêtées à l'aéroport d'Antalya avec de l'or caché au fond de leur vagin.

Ajoutons que d'un point de vue plus scatologique, les personnages traînent dans leurs excréments : on interdit à la petite fille de Mères sans chatte et à celle d'A(II)R $h+$ d'aller $^{\prime}$ aux toilettes en dehors des cours et elles finissent par faire sur elles; selon la jeune femme de FUCK YOU, Eu.ro.Pa!, « en Union soviétique, le sexe n'existait pas! Une sorte de menstruation continue.»(17) Esinencu évoque encore dans ce monologue le manque d'eau courante depuis trois ans dans les appartements et raconte que «les gens jettent des sacs remplis de merde par les fenêtres de leurs immeubles. » (26)

C'est la merde en Moldavie et Esinencu décrit cette merde. Et si elle la balance « dans la gueule » de ses concitoyens à coups de mots provocants, elle en fait de même en s'en prenant à l'Europe, à l'Union Européenne, qui a sa part de responsabilité dans le marasme moldave. Je traite dans un article intitulé « Le sexe est dans le titre » de trois pièces roumaine, moldave, macédonienne, de Dejan Dukovski, Peca Stefan et Esinencu. Les trois titres de leurs pièces sont provocateurs et grossiers: Quel est l'enfoiré qui a commencé le premier?, Uncle Fucker et FUCK YOU, Eu.ro.Pa!, et disent en substance la même chose, à savoir ils demandent à l'Europe de retourner dans le con de sa mère. Si les auteurs du Kitchen-sink theater et du In-yer-face drama révèlent les maladies sociales de leur propre pays, le propre des auteurs d'Europe centrale et orientale est de rendre l'Europe responsable d'un état de fait affligeant. La politique de leurs pays est en lien direct avec le passage du communisme au système capitaliste et à ses tares, un système qui a creusé le gouffre entre les plus riches et les plus pauvres. Les jeunes auteurs «Dans ta gueule" se font les porte-parole de leur génération perdue pour qui le passage à l'Europe n'a pas été aussi bénéfique qu'espéré. Esinencu rejette à son tour l'idée fausse de la grande Europe et nous avoue avoir peur "de l'idée de l'Union. N'importe laquelle. Peut-être à cause de l'Union soviétique. » Dans FUCK YOU, Eu.ro.pa!, son personnage a rêvé longtemps de l'Europe et sa déception est grande quand enfin elle y parvient à l'âge adulte. A la joie de l'éradication du bloc communiste totalitaire, «l'Union soviétique n'existait plus. Papa était heureux» (21), succède la découverte 
d'une Europe vide pour cause de trop plein : au rien succède la surconsommation outrancière et la nausée.

\section{Un désir de re-construction littéraire et politique}

La dramaturge se met face à son public moldave pour le provoquer haut et fort d'un ton violent, impudique, imprudent, et l'accuse de tous les maux. En vérité, selon l'étymologie du verbe latin pro-vocare, tout comme le faisaient les auteurs britanniques des années 80 , elle met à mal ce public et l'appelle « en avant ", dans le sens où elle le conjure d'aller de l'avant. Elle l'«appelle dehors" de lui-même, elle le conjure de changer, de se construire autrement. Dans FUCK YOU, EU.ro.Pa!, le personnage insulte une voisine en roumain: "Du-te în pizda ma-tiiiii !» (19), l'enjoignant de retourner dans le con de sa mère. Que les vagins, qui ne servent qu'à transporter de l'or turc et à annihiler les enfants en les avalant, expulsent enfin des hommes nouveaux et que chacun re-naisse par la littérature, tel semble être le souhait ésinencien : elle appelle l'homme nouveau, un peu comme les communistes en leurs temps. Avec sa compagnie Spalatorie, "Pressing» en français, l'artiste veut former les esprits, laver le linge sale pour lui rendre des couleurs moins nauséabondes. De l'évier de la cuisine ${ }^{2}$ au lave-linge, il n'y avait qu'un pas à franchir, qu'Esinencu franchit grâce à la force de sa langue. La violence et l'outrance des situations qu'elle dépeint sont contrebalancées par un désir de construction littéraire et dramaturgique original.

Le langage créé par l'auteur semble formellement déstructuré, haché, comme sa patrie ; il se présente sous la forme de phrases très courtes, presque des vers visuellement :

maman

la sœur de maman

la grand'mère

la voisine

le docteur [...]

ma prof de biologie

et la bibliothécaire

la confiture

on la met en bocaux en été

pour la manger en hiver (1)

Cet incipit de Mères sans chatte est typique de l'écriture d'Esinencu : le texte peut se dire de manière syncopée ou d'une seule traite filée. Au lecteur de reconstruire la phrase, le phrasé, de faire un choix de respiration, de ponctuation ; il est ainsi amené à un acte créateur grâce à cette parole qu'il a lui-même à sculpter, à moduler.

Par ailleurs, le lecteur et le spectateur ésinencien participent à l'écriture, à sa mise en scène, plutôt sa mise en voix du fait que la dramaturge qui écrit un monologue convoque en vérité plusieurs voix du passé par le biais du seul personnage. Les paroles rapportées par lui sont intégrées dans sa logorrhée non ponctuée, sans guillemets, sans espace, elles se fondent dans son propre discours; la difficulté vient du fait qu'elles le sont à la fois au discours direct, indirect et indirect libre. Au lecteur, au spectateur de rendre sa parole à l'émetteur :

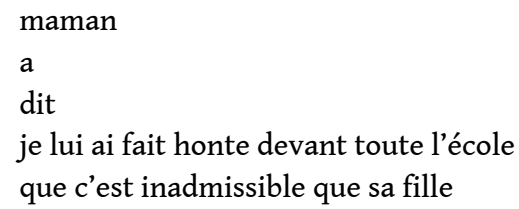


apparaisse en pleine rue

en salopette

que

\section{SEULSLESGARCONSPORTENTDESPANTALONS}

a-t-elle dit encore

et qu'est-ce qu'elle va faire maintenant?

comment j'irais à l'école maintenant?

comment affronter le regard des autres mères ? (7)

Esinencu construit enfin sur le déluge par sa dramaturgie basée sur le leitmotiv et la récurrence de motifs. Certes, l'Histoire se répète et des tirades cycliques reviennent, mais il faut les aborder différemment pour faire évoluer l'humain. Dans FUCK YOU, Eu.ro.Pa!, le personnage reprend très régulièrement la même phrase «Papa, il faut que je te dise quelque chose »; dans Mères sans chatte, la gamine répète en un leitmotiv inquiétant comme une phrase apprise par cœur sur la meilleure façon de se tenir selon la norme : « la confiture on la met en bocaux en été pour la manger en hiver, les gros concombres en hiver, les bananes en été et en hiver "; la fillette évoque une situation différente avec une femme nouvelle, mais à chaque fois, il s'agit d'une scène où la femme en question nie la sexualité féminine. Dans $A(I I) R h+$, les scènes de réactions racistes du protagoniste reviennent à intervalles réguliers. Autour de ces résonances, la fable avance, le personnage se construit dans une folie qui le mène au paroxysme de l'absurde et de la violence ; au public de comprendre qu'il faut nier et rompre le cycle monomaniaque pour envisager une autre fin que la mort. Dans FUCK You, Eu.ro.pa!, les passages liminaires sont une liste de maladies et une liste de médicaments : le théâtre ésinencien diagnostique et peut soigner.

On trouve des parallèles évidents entre les dramaturges du Kitchen-sink, du In-yer-face, puis du « Dans ta gueule » d'Europe centrale et balkanique, sans qu'il y ait de filiation volontaire : chez ces artistes en colère, qui ont une sombre vision de l'homme et de notre société, «le drame sur la scène est remplacé par le drame du monde » (2) écrit Miloš Lazin dans son article intitulé "Le drame d'un nouveau monde; l'écriture théâtrale à l'époque de la mondialisation totale ", dans lequel il déplore la fin de l'utopie des Lumières. Pourtant le nouveau théâtre, éminemment politique, par la violence de ses images crues et d'un langage obscène exerce un pouvoir cathartique : empathique et exutoire, il est aussi éclairant et porteur d'espoir, puisqu'il vise à susciter une métamorphose du citoyen. Nicoleta Esinencu fait elle aussi le choix de l'ultra-violence pour dénoncer les dérives sociétales nationales et européennes. Elle pratique ce «tableau clinique des violences" (13) qu'évoque Jean-Marc Lantéri ou comme le disait Sarah Kane elle-même "l'anatomie du mal " (Stephenson, Langridge, 197). Mais cette provocation est-elle efficace pour atteindre un public rétrograde? Assurément pas en Moldavie. Politiquement incorrecte, Esinencu provoque, mais surtout provoque des réactions de rejet d'un auditoire et d'institutions qui n'aiment pas être malmenées. J'ai assisté à Chisinau à un débat-pugilat, où l'auteur était attaquée sur ses positions : elle incarne à Chisinau une image d'avant-garde théâtrale non reconnue, une image sulfureuse détestable et détestée. Idéalement Esinencu veut laver le linge sale avec Spalatorie et donner naissance à une nouvelle Moldavie; concrètement, elle donne à entendre son théâtre considéré comme underground dans une cave du centreville, à deux pas du Théâtre National, qui propose d'éternels classiques et des spectacles folkloriques. Esinencu est appréciée et publiée en Allemagne et en France, en Roumanie, pas en Moldavie ${ }^{3}$. Controversée là-bas, elle est pourtant une grande dramaturge et son écriture vaut d'être louée par sa nouveauté, son intelligence, sa 
subtilité, la force de l'horreur et des tabous démontés... si la Moldavie ne l'a pas encore compris, l'Europe est désormais à l'écoute, où Esinencu est reconnue comme auteur et metteur en scène, « mazarfack !» (15).

\section{BIBLIOGRAPHIE}

Dolmieu, Dominique. Entretien par courriel en janvier 2013.

Dukovski, Dejan. Quel est l'enfoiré qui a commencé le premier ?, traduit du macédonien par

Harita Wybrands, Paris : L'Espace d'un instant, 2002.

Esinencu, Nicoleta. FUCK YOU, Eu.ro.Pa!, traduit du roumain par Mirella Patureau, Paris : L'Espace d'un instant, 2006.

--. A(II)Rh+, traduit du roumain par Mirella Patureau, tapuscrit.

--. Mères sans chatte, traduit du roumain par Mirella Patureau, tapuscrit.

-—. Entretien par courriel en 2010.

Lanteri, Jean-Marc. "L'Age d'or et la Dame de fer", in Ecritures contemporaines 5,

Dramaturgies britanniques (1980-2000). Paris-Caen : Lettres modernes Minard,, 2002.

Lazin, Miloš. « Le drame d'un nouveau monde », article interne à la Maison d'Europe et d'Orient.

-—. «A quoi tient le succès de Biljana Srbljanovic ? », Revue des études slaves, t. LXXVII, fasc. 1-2, Institut d'études slaves, 2006.

Patureau, Mirella. Entretien par courriel en janvier 2013.

Stefan, Peca. U.F., traduit du roumain par Gabriel Marian et Laurent Muhleisen, Montpellier : Editions Climats et Maison Antoine Vitez, 2004.

Stephenson, Heidi, et Langridge, Natasha. "La forme est le sens, entretien avec Sarah Kane", in La Forme est le sens, in LEXI/textes 3. Paris : Théâtre National de la Colline, L’Arche,1999.

\section{NOTES}

1. Entretien électronique NE-VB, 2010 : «Ce n'est pas un texte autobiographique. C'est plutôt la biographie de ma génération, le sentiment de ma génération. »

2. The kitchen-sink drama : le théâtre de l'évier de la cuisine.

3. Pour ce qui est des publications en Roumanie, on n'y trouve que A(II)RH+, chez l'éditeur "Idea" de Cluj-Napoca. 


\section{RÉSUMÉS}

La dramaturge moldave Nicoleta Esinencu est l'auteur «Dans ta gueule » de textes aux titres et au langage provocateurs. Elle dénonce brutalement les actions de ses concitoyens sous le communisme et leurs comportements politiques contemporains. Dans ses trois monologues FUCK YOU, Eu.ro.Pa!, A (II) Rh+, Mères sans chatte, la critique familiale glisse vers une accusation citoyenne nationale. Pourtant, si Esinencu pro - voque ses compatriotes, elle les met à mal et les appelle « en avant » dans le sens où elle les conjure d'aller de l'avant ; étymologiquement elle les " appelle dehors » d'eux-mêmes pour se construire autrement : les situations outrancières et la violence dont elle témoigne sont contrebalancées par une construction littéraire et dramaturgique originale de ses monologues censés inciter le public à la création d'un homme moldave nouveau.

The moldavian playwright, Nicoleta Esinencu, is the author of "Dans ta gueule " texts with provoking titles and language abuse. She violently denounces the acts of her fellow citizens under the communist regime and their current political behaviours. In her three monologues FUCK YOU, Eu.ro.Pa!, A (II) Rh+, Mères sans chatte, the family satire drifts towards a national patriotic criticism. Although Esinencu provokes her fellow citizens, she ill-treats them and calls them "forwards" in the sense that she entices them to move forwards: etymologically speaking she "calls them out" of themselves so as to build themselves otherwise: the exaggerated situations and the violence she resorts to are balanced-off by an original literary and theatrical construction of her monologues supposed to entice the public to create a new moldavian man.

\section{INDEX}

Mots-clés : Dans ta gueule, Esinencu, In-yer-face, Moldavie, monologue, théâtre

Keywords : Esinencu, In-yer-face, Moldavia, monologue, theatre

\section{AUTEUR}

\section{VÉRONIQUE BOUTIN}

chercheuse indépendante

veroniqueboutin.ee@gmail.com 